\title{
Static and Dynamic Scaling Relations for Earthquakes and Their Implications for Rupture Speed and Stress Drop
}

\author{
by Hiroo Kanamori and Luis Rivera
}

\begin{abstract}
We investigate the relation between a static scaling relation, $M_{0}$ (seismic moment) versus $f_{0}$ (spectral corner frequency), and a dynamic scaling relation between $M_{0}$ and $E_{\mathrm{R}}$ (radiated energy). These two scaling relations are not independent. Using the variational calculus, we show that the ratio $\tilde{e}=E_{\mathrm{R}} / M_{0}$ has a lower bound, $\tilde{e}_{\text {min }}$, for given $M_{0}$ and $f_{0}$. If the commonly used static scaling relation $\left(M_{0} \propto f_{0}^{-3}\right)$ holds, then $\tilde{e}_{\text {min }}$ must be scale independent and should not depend on the magnitude, $M_{\mathrm{w}}$. The observed values of $\tilde{e}$ for large earthquakes [e.g., $\left.\tilde{e}\left(M_{\mathrm{w}} 7\right)\right]$ are close to $\tilde{e}_{\min }$. The observed values of $\tilde{e}$ for small earthquakes are controversial, but the reported values of $\tilde{e}\left(M_{\mathrm{w}} 3\right)$ range from 1 to 0.1 of $\tilde{e}\left(M_{\mathrm{w}} 7\right)$, suggesting that $\tilde{e}_{\min }$ may decrease as $M_{\mathrm{w}}$ decreases. To accommodate this possibility, we need to modify the $M_{0}$ versus $f_{0}$ scaling relation to $M_{0} \propto f_{0}^{-(3+\varepsilon)}(\varepsilon \leq 1)$, which is allowable within the observational uncertainties. This modification leads to a scale-dependent $\tilde{e}_{\min }, \tilde{e}_{\min } \propto$ $10^{1.5 M_{\mathrm{w}} \varepsilon /(3+\varepsilon)}$, and a scale-dependent $\Delta \sigma_{\mathrm{s}} V^{3}\left(\Delta \sigma_{\mathrm{s}}=\right.$ static stress drop, $V=$ rupture speed), $\Delta \sigma_{\mathrm{s}} V^{3} \propto 10^{1.5 M_{\mathrm{w}} \varepsilon /(3+\varepsilon)}$, and it can accommodate the range of presently available data on these scaling relations. We note that the scaling relation, $\Delta \sigma_{\mathrm{s}} V^{3} \propto$ $10^{1.5 M_{\mathrm{w}} \varepsilon /(3+\varepsilon)}$, suggests that even if $\tilde{e}$ is scale independent and $M_{0} \propto f_{0}^{-3}$ (i.e., $\varepsilon=0$ ), $\Delta \sigma_{\mathrm{s}}$ is not necessarily scale independent, although such scale independence is often implied. Small and large earthquakes can have significantly different $\Delta \sigma_{\mathrm{s}}$ and $V$; if $\tilde{e}$ varies with $M_{\mathrm{w}}$, as suggested by many data sets, the difference can be even larger, which has important implications for rupture physics.
\end{abstract}

\section{Introduction}

The relation between seismic moment, $M_{0}$, and a length scale, $\tilde{L}$ (e.g., square root of the rupture area, rupture length, etc.), of earthquakes has been widely used in seismology as a useful gross scaling relation between static parameters. For small earthquakes, it is difficult to determine the rupture length, and the pulse width, $T$, or the spectral corner frequency, $f_{0}(\propto 1 / T)$, is commonly used as a proxy for the source dimension. The most commonly used scaling relation is $M_{0} \propto f_{0}^{-3}$ (Brune, 1970, 1971; Hanks and Wyss, 1972; Abercrombie and Leary, 1993; Boatwright, 1994).

On the other hand, the relation between the radiated energy, $E_{\mathrm{R}}$, and the seismic moment, $M_{0}$, of an earthquake can be considered a dynamic scaling relation because the radiated energy reflects the dynamics of faulting. In practice, the ratio $E_{\mathrm{R}} / M_{0}$ has long been used in seismology as a useful parameter that characterizes the dynamic properties of an earthquake (Aki, 1966; Wyss and Brune, 1968). The ratio is usually multiplied by rigidity, $\mu$, and is called the "apparent stress." The ratio can be written as $\tilde{e}=\left(E_{\mathrm{R}} / M_{0}\right)=(1 / \mu)$ $(1 / D)\left(E_{\mathrm{R}} / S\right)(D$, fault offset; $S$, fault area) and can be inter- preted as being proportional to the energy radiated per unit area and per unit slip. In view of its importance for understanding the dynamic characters of earthquakes, many studies have been devoted to determination of $\tilde{e}$. Unfortunately, it is difficult to determine $E_{\mathrm{R}}$ accurately because of the complex wave propagation effects in the Earth, especially for small earthquakes, and the results were widely scattered. In many studies, $\tilde{e}$ is found to decrease as the magnitude, $M_{\mathrm{w}}$, decreases. However, because of the large uncertainties in the measurements, whether $\tilde{e}$ is scale independent or not has been vigorously debated (e.g., Ide and Beroza, 2001), and the problem remains unresolved.

In this article, we investigate the static and dynamic scaling relations together. We will show that these two scaling relations are not independent, and, regardless of whether $\tilde{e}$ is scale independent or not, a combined use of these relations provides useful constraints on the $\tilde{e}$ versus $M_{\mathrm{w}}$ relation, $M_{0}$ versus $f_{0}$ relation, rupture speed $(V)$, and static stress drop $\left(\Delta \sigma_{\mathrm{s}}\right)$ for small earthquakes; $V$ and $\Delta \sigma_{\mathrm{s}}$ are key parameters for understanding the rupture physics. 


\section{Energy-Moment Relation}

Recent improvements in data quality and methodology have significantly improved the accuracy of $E_{\mathrm{R}}$ determination for large earthquakes (e.g., $M_{\mathrm{w}}>6$ ) (e.g., Boatwright and Choy, 1986; Boatwright et al., 2002; Venkataraman et $a l ., 2002)$. For small earthquakes, it is still difficult to correct for the propagation effects. Nevertheless, using down-hole instruments, or with careful removal of path effects, large amounts of high-quality data for small earthquakes have accumulated (Abercrombie, 1995; Mayeda and Walter, 1996; Izutani and Kanamori, 2001; Prejean and Ellsworth, 2001; Kinoshita and Ohike, 2002). The ratio depends on many seismogenic properties of the source region so that it varies significantly for earthquakes in different tectonic environments, such as continental crust, subduction zone, deep seismic zone, and so on (Choy and Boatwright, 1995; PerezCampos and Beroza, 2001; Venkataraman and Kanamori, unpublished manuscript). However, the data for the same type of earthquakes exhibit an interesting trend. Figure 1 shows the results for crustal earthquakes in California and Japan. Taken at face value, despite the large scatter, the ratio $\tilde{e}$ decreases as the magnitude, $M_{\mathrm{w}}$, decreases. For large earthquakes $\left(M_{\mathrm{w}} \approx 7\right), \tilde{e}$ is approximately $5 \times 10^{-5}$; but it is approximately a factor of 10 smaller at $M_{\mathrm{w}} \approx 3$ and a factor of 100 smaller at $M_{\mathrm{w}} \approx 1$. Results for even smaller earthquakes show even smaller values of $\tilde{e}$ (e.g., Jost et al., 1998; Richardson and Jordan, 2002). Ide and Beroza (2001) suggested that many of the published $\tilde{e}$ versus $M_{\mathrm{w}}$ relations could be biased in a similar way (i.e., decrease in $\tilde{e}$ for small events), because of the inadequate correction for path effects or the limited instrumental passband, and $\tilde{e}$ could be scale independent.

We note that, in principle, both $M_{0}$ and $E_{\mathrm{R}}$ are computed from the same seismograms of an earthquake and are not completely independent. Thus, the extremely large variation of $\tilde{e}$ with $M_{\mathrm{w}}$ shown in Figure 1 is somewhat surprising. Most commonly, especially for small earthquakes, far-field $P$ or $S$ waves are used (e.g., Abercrombie, 1995). In the commonly used methods of $M_{0}$ and $E_{\mathrm{R}}$ determinations, all the propagation effects (e.g., radiation pattern, geometrical spreading factor, attenuation, receiver and source structure effects) have been removed, so that the problem is reduced to that in a homogeneous whole space. Since the most energy is in $S$ waves, we consider only $S$ waves.

Let $u(t)$ be the radiation pattern-corrected far-field displacement. For an earthquake (i.e., double couple), $u(t)$ is a one-sided pulse, not necessarily of a simple waveform, with a total duration, $T$, with $u(0)=u(T)=0$. Then the seismic moment, $M_{0}$, which is proportional to the area under $u(t)$, is given by

$$
M_{0}=C_{\mathrm{M}} \int_{0}^{T} u(t) d t
$$

where $C_{\mathrm{M}}=4 \pi \rho r \beta^{3}$, and the radiated energy $E_{\mathrm{R}}$ is given by

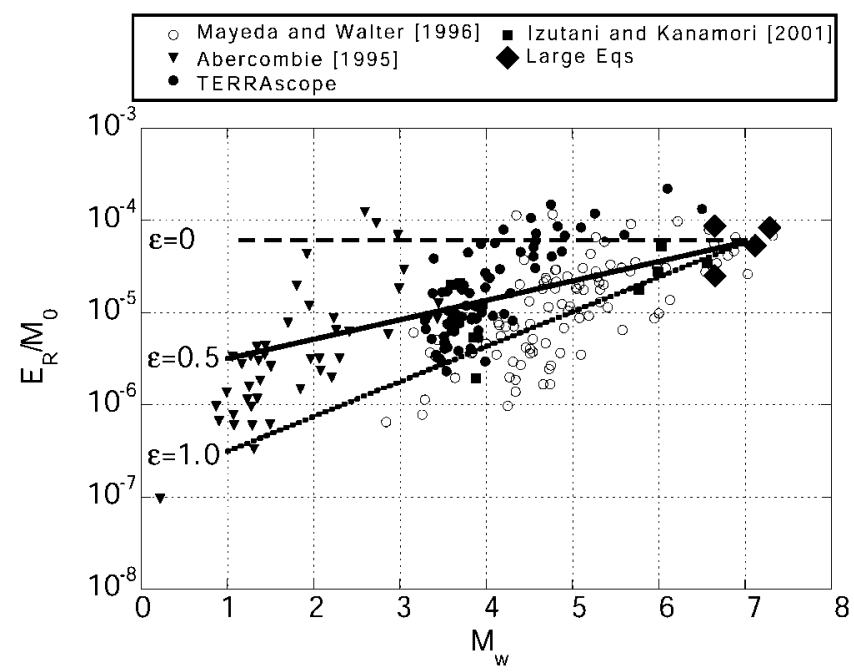

Figure 1. Energy-to-moment ratio, $\tilde{e}=E_{\mathrm{R}} / M_{0}$, for shallow crustal earthquakes in California and Japan. The dashed line is the trend of $\tilde{e}_{\min }$ for the commonly used scaling relation $M_{0} \propto f_{0}^{-3}$. Solid and dotted lines show, respectively, the expected trends of $\tilde{e}_{\min }$ for the two cases $(\varepsilon=1.0$ and $\varepsilon=0.5)$ of the modified scaling relation between $M_{0}$ and the corner frequency, $f_{0}$, given by equation (12). Data sources: Abercrombie (1995), Mayeda and Walter (1996), Izutani and Kanamori (2001), and Kanamori et al. (1993). Data for large earthquakes: Mayeda and Walter (1996), Kanamori and Heaton (2000), McGarr and Fletcher (2002), Boatwright et al. (2002), Venkataraman et al. (2002), and Izutani and Kanamori (2001).

$$
E_{\mathrm{R}}=C_{\mathrm{E}} \int_{0}^{T} \dot{u}^{2}(t) d t
$$

where $C_{\mathrm{E}}=8 \pi \rho r^{2} \beta / 5$ (e.g., Haskell, 1964), where $\beta$, $\rho$, and $r$ are the $S$ velocity, density, and distance from the source, respectively. Any $u(t)$, as long as its time integral over $T$ is the same, gives the same seismic moment, $M_{0}$. However, because $u(t)$ can have any time derivative $\dot{u}(t), E_{\mathrm{R}}$ is not uniquely determined, even if $M_{0}$ is fixed. If $u(t)$ varies very rapidly, $E_{\mathrm{R}}$ can be very large; in fact it can be unbounded [e.g., for a boxcar $u(t)$ ]. However, for given $M_{0}$ and $T, E_{\mathrm{R}}$ has a lower bound, as shown below.

This problem can be formulated using the variational calculus. We try to determine the minimum value of $E_{\mathrm{R}}$ with the constraint that $M_{0}$ is given. Introducing the Lagrangian multiplier $\lambda$, this is equivalent to minimizing the functional

$$
J(u, \dot{u})=E_{\mathrm{R}}-\lambda M_{0}=C_{\mathrm{E}} \int_{0}^{T} \dot{u}^{2}(t) d t-\lambda C_{\mathrm{M}} \int_{0}^{T} u(t) d t .
$$

The Euler equation for $\delta J=0$ is

$$
\left(\frac{\partial}{\partial u}-\frac{\mathrm{d}}{d t} \frac{\partial}{\partial \dot{u}}\right)\left[C_{\mathrm{E}} \dot{u}^{2}-\lambda C_{\mathrm{M}} u\right]=0,
$$


from which we obtain $\ddot{u}=-\lambda C_{\mathrm{M}} / 2 C_{\mathrm{E}}$. Integrating this twice and using the conditions $u(0)=u(T)=0$, we obtain

$$
u(t)=\frac{\lambda}{4} \frac{C_{\mathrm{M}}}{C_{\mathrm{E}}} t(T-t)
$$

Substituting equation (5) in equation (1), the Lagrangian multiplier $\lambda$ can be determined as

$$
\lambda=\frac{24 C_{\mathrm{E}} M_{0}}{C_{\mathrm{M}}^{2} T^{3}}
$$

Substituting equation (6) in equation (5), we finally obtain

$$
u(t)=\frac{6 M_{0}}{C_{\mathrm{M}} T^{3}} t(T-t)
$$

and

$$
\tilde{e}_{\min } \equiv\left(\frac{E_{\mathrm{R}}}{M_{0}}\right)_{\min }=\frac{12 C_{\mathrm{E}}}{C_{\mathrm{M}}^{2} T^{3}} M_{0}=\frac{6}{5 \pi \mu}\left(\frac{V}{\beta}\right)^{3} \frac{M_{0}}{(V T)^{3}},
$$

where $V$ is the rupture speed. It can be shown that $\tilde{e}_{\text {min }}$ given above is indeed a minimum and the displacement that gives $\tilde{e}_{\min }$ is a symmetric parabola, and any departure from it increases the ratio.

If the length scale of the source is $\tilde{L}, M_{0} \approx \Delta \sigma_{\mathrm{s}} \tilde{L}^{3} \approx$ $\Delta \sigma_{\mathrm{s}}(V T)^{3}$, where $\Delta \sigma_{\mathrm{s}}$ is the static stress drop. For a circular fault model, which is commonly used for small earthquakes, $\tilde{L}$ is taken to be the radius, and $M_{0}=(16 / 7) \Delta \sigma_{\mathrm{s}} \tilde{L}^{3}=(16 /$ 7) $\Delta \sigma_{\mathrm{s}}(V T)^{3}$ (e.g., Brune, 1970). Then equation (8) can be written as

$$
\tilde{e}_{\min } \approx 0.87\left(\frac{V}{\beta}\right)^{3}\left(\frac{\Delta \sigma_{\mathrm{s}}}{\mu}\right)=0.87\left(\frac{1}{\mu \beta^{3}}\right)\left(\Delta \sigma_{\mathrm{s}} V^{3}\right) .
$$

For large crustal earthquakes, using $\mu=30 \mathrm{GPa}, \Delta \sigma_{\mathrm{s}}=3$ $\mathrm{MPa}$, and $(V / \beta)=0.9$, we have $\tilde{e}_{\min }=6.3 \times 10^{-5}$, which is close to the average value for large earthquakes shown in Figure 1. Although there is no obvious physical reason why the observed ratio $\tilde{e}$ should be close to the minimum value, this is an interesting result. Because equation (9) gives the minimum value, the observed values must be larger than that computed from equation (9). Yet, the observed values of $\tilde{e}$ for small earthquakes are 1-2 orders of magnitude smaller than the $\tilde{e}_{\text {min }}$ expected for the conventional values of $\Delta \sigma_{\mathrm{s}}$ and $V$ (Fig. 1). It may appear from equation (9) that either reducing $V$ or $\Delta \sigma_{\mathrm{s}}$ or both for small earthquakes can explain this situation. However, as shown below, we cannot reduce $\Delta \sigma_{\mathrm{s}}$ or $V$ arbitrarily because of the scaling relation between $M_{0}$ and $f_{0}$.

\section{Moment-to-Corner-Frequency Scaling Relation}

Many investigators studied the scaling relation between $M_{0}$ and the spectral corner frequency, $f_{0}$. Estimation of the corner frequency for small earthquakes is difficult because the measurements are severely hampered by the attenuation along the path and the limited passband of the seismograph used. Nevertheless, the results obtained by Abercrombie (1995) using the down-hole instruments are considered among the most reliable. As shown in Figure 2, Abercrombie's (1995) results, as well as many others' (Hanks and Wyss, 1972; Abercrombie and Leary, 1993; Boatwright, 1994; Kinoshita and Ohike, 2002), are consistent with the model suggested by Brune (1970) and are given by

$$
M_{0} \propto f_{0}^{-3} .
$$

From a physical scaling relation, $M_{0} \approx \Delta \sigma_{\mathrm{s}} \tilde{L}^{3} \approx \Delta \sigma_{\mathrm{s}}(V T)^{3}$ $\approx\left(\Delta \sigma_{\mathrm{s}} V^{3}\right) f_{0}^{-3}$, we can write

$$
M_{0}=C_{1}\left(\Delta \sigma_{\mathrm{s}} V^{3}\right) f_{0}^{-3} .
$$

Comparison of this physical scaling relation and the empirical relation (10) suggests that $\Delta \sigma_{\mathrm{s}} V^{3}$ is constant, or scale independent. Then, equation (9) means that $\tilde{e}_{\min }$ must be scale independent, which contradicts the result shown in Figure 1, because the observed values of $\tilde{e}$ for small earthquakes are smaller than $\tilde{e}_{\min }$ given by equation (9) for large earthquakes. Thus, we must conclude that the small values of $\tilde{e}$ shown in Figure 1 and the empirical scaling relation (10) are mutually exclusive. We can vary both $V$ and $\Delta \sigma_{\mathrm{s}}$, but because $\Delta \sigma_{\mathrm{s}} V^{3}$ is constrained to be constant (equations 10 and $11), \tilde{e}_{\min }$ that is determined by the combination, $\Delta \sigma_{\mathrm{s}} V^{3}$ (equation 9), is scale independent.

\section{Alternative Scaling Relation and Constraints on Rupture Speed and Stress Drop}

The conclusion we reached above is that (1) if the scaling $M_{0} \propto f_{0}^{-3}$ holds, then $\tilde{e}$ must be equal to, or larger than,

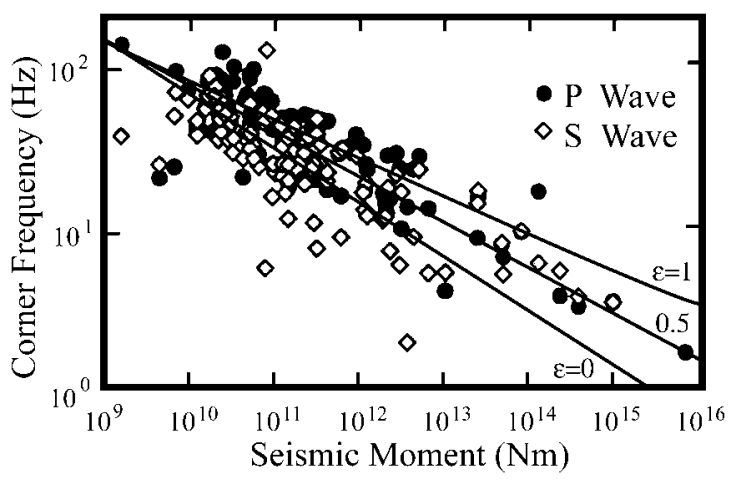

Figure 2. Moment versus corner frequency relation (Abercrombie, 1995). The solid lines show the relation $M_{0} \propto f_{0}^{-(3+\varepsilon)}$. 
$\tilde{e}_{\text {min }}$ estimated for large earthquakes, or (2) if $\tilde{e}$ is scale dependent as suggested in Figure 1, then we cannot have $M_{0}$ $\propto f_{0}^{-3}$ scaling. In case 2 , we need to modify the scaling relation (10). Although many studies have suggested the relation $M_{0} \propto f_{0}^{-3}$, the scatter of the data is very large, and the data can accommodate a slightly different scaling relation. For example, we can assume, instead of equation (10), a scaling relation

$$
M_{0}=C_{2} f_{0}^{-(3+\varepsilon)}
$$

where $\varepsilon$ is a small $(\leq 1)$ constant. In fact, this kind of scaling relation has been suggested by several investigators (e.g., Xie and Patton, 1999; Izutani and Kanamori, 2001).

We now investigate the consequence of this scaling relation and its implications. In this case, from equations (11) and (12), we have

$$
\Delta \sigma_{\mathrm{s}} V^{3}=\frac{C_{2}}{C_{1}} f_{0}^{-\varepsilon} \propto M_{0}^{\varepsilon / 3+\varepsilon}
$$

Thus, from equation (9),

$$
\tilde{e}_{\min } \propto M_{0}^{\varepsilon / 3+\varepsilon}
$$

and $\tilde{e}_{\text {min }}$ becomes scale dependent, if $\varepsilon>0$. For example, if we take the value at $M_{\mathrm{w}} 7, \tilde{e}_{\min }\left(M_{\mathrm{w}} 7\right)$, as a reference, then

$$
\frac{\tilde{e}_{\min }\left(M_{\mathrm{w}}\right)}{\tilde{e}_{\min }\left(M_{\mathrm{w}} 7\right)}=10^{1.5\left(M_{\mathrm{w}}-7\right) \varepsilon /(3+\varepsilon)}
$$

and

$$
\left[\frac{V\left(M_{\mathrm{w}}\right)}{V\left(M_{\mathrm{w}} 7\right)}\right]^{3}=\left[\frac{\Delta \sigma_{\mathrm{s}}\left(M_{\mathrm{w}}\right)}{\Delta \sigma_{\mathrm{s}}\left(M_{\mathrm{w}} 7\right)}\right]^{-1} 10^{1.5\left(M_{\mathrm{w}}-7\right) \varepsilon /(3+\varepsilon)}
$$

Equation (15) gives the dependence of $\tilde{e}_{\min }$ on $M_{\mathrm{w}}$; the trend is shown in Figure 1 . The case with $\varepsilon=0.5$ yields an adequate trend for events $M_{\mathrm{w}}>3$. As we can see in Figure 2, the $M_{0}$ versus $f_{0}$ scaling relation with $\varepsilon=0.5$ is well within the scatter of the data and is acceptable.

Then, the next question is what the implication of this is for $V$ and $\Delta \sigma_{\mathrm{s}}$. Figure 3 shows the relation (16) for events with $M_{\mathrm{w}} 3$ and $\varepsilon=0$ (dashed line) and $\varepsilon=0.5$ (solid line). If we assume that the observed trend of $\tilde{e}$ is an artifact, then $\varepsilon=0$. Then $V$ and $\Delta \sigma_{\mathrm{s}}$ for $M_{\mathrm{w}} 3$ events can take any value given by the dashed line. In contrast, if we accept the observed trend of $\tilde{e}$, and use $\varepsilon=0.5$, then $V$ and $\Delta \sigma_{\mathrm{s}}$ for $M_{\mathrm{w}}$ 3 events can take any value given by the solid line. For illustration purposes, we consider the following three cases:

1. If $\varepsilon=0$ and $\Delta \sigma_{\mathrm{s}}$ for $M_{\mathrm{w}} 3$ events is 10 times larger than that for $M_{\mathrm{w}} 7$ events, then $V$ for $M_{\mathrm{w}} 3$ events must be 0.43 times that for $M_{\mathrm{w}} 7$ events (marked by an inverted triangle in Fig. 3). This means that even if both the com-

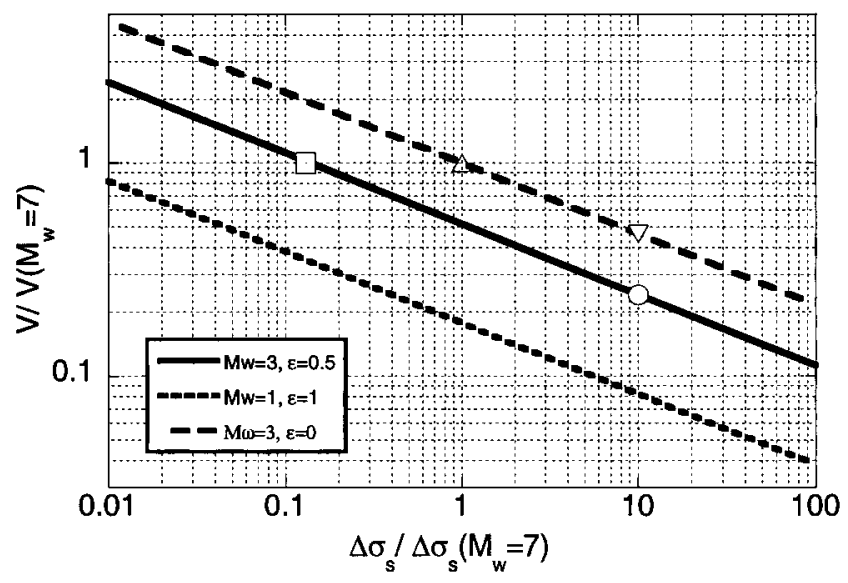

Figure 3. The relation between relative rupture speed $V\left(M_{\mathrm{w}}\right) / V\left(M_{\mathrm{w}} 7\right)$ and relative stress drop $\Delta \sigma_{\mathrm{s}}\left(M_{\mathrm{w}}\right) / \Delta \sigma_{\mathrm{s}}\left(M_{\mathrm{w}} 7\right)$. The dashed line is for $M_{\mathrm{w}} 3$ and $\varepsilon=0$. The solid line is for $M_{\mathrm{w}} 3$ and $\varepsilon=0.5$, and the dotted line is for $M_{\mathrm{w}} 1$ and $\varepsilon=1.0$. The inverted triangle and circle indicate cases 1 and 2 , respectively, and the square indicates case 3 discussed in the text. The triangle indicates the case in which $\Delta \sigma_{\mathrm{s}}$ and $V$ are scale independent.

monly used scaling relation $M_{0} \propto f_{0}^{-3}$ and a scaleindependent $\tilde{e}$ hold, $V$ and $\Delta \sigma_{\mathrm{s}}$ are not necessarily the same between small and large earthquakes.

2. If $\varepsilon=0.5$ and $\Delta \sigma_{\mathrm{s}}$ for $M_{\mathrm{w}} 3$ events is 10 times larger than that for $M_{\mathrm{w}} 7$ events, then $V$ for $M_{\mathrm{w}} 3$ events must be 0.23 times that for $M_{\mathrm{w}} 7$ events (marked by a circle in Fig. 3).

3. If $\varepsilon=0.5$ and $V$ for $M_{\mathrm{w}} 3$ events is the same as that for $M_{\mathrm{w}} 7$ events, then $\Delta \sigma_{\mathrm{s}}$ for $M_{\mathrm{w}} 3$ events must be approximately a factor of 10 smaller than that for $M_{\mathrm{w}} 7$ events (marked by a square in Fig. 3).

Other combinations are of course possible. Because very few observations for $V$ and $\Delta \sigma_{\mathrm{s}}$ are available for small earthquakes, we cannot resolve this problem now. However, these cases have very different and interesting implications. In cases 1 and 2, the implication is that small earthquakes represent failures of small-scale asperities with high stress concentration. Nadeau and Johnson (1998) suggested high stress drops for very small earthquakes in Parkfield, California. The slow rupture speed suggests that the fracture energy is large relative to the radiated energy (Kostrov, 1966; also see Kanamori et al., 1998). Because the ratio of fracture energy to radiated energy controls the dynamics of rupture, this means that small and large earthquakes are dynamically different. In contrast, in case 3, small and large earthquakes are dynamically similar (i.e., $V=$ constant), but small earthquakes are simply low-stress-drop events. This is similar to the case suggested by Beeler et al. (2003). The resolution of this problem must await accumulation of more reliable data on $V$ and $\Delta \sigma_{\mathrm{s}}$ for small earthquakes.

A similar argument could be made for even smaller 
$\left(M_{\mathrm{w}}<3\right)$ earthquakes (dotted line in Fig. 3), but the uncertainties in the measurements of $\tilde{e}$ may be too large to warrant any more detailed analysis at present. However, to explain the extremely small values of $\tilde{e}$ for very small $\left(M_{\mathrm{w}}<3\right)$ earthquakes, we would need to modify the $M_{0}$ versus $f_{0}$ relation, $\Delta \sigma_{\mathrm{s}}$, and $V$ very substantially from the commonly used relation or values.

\section{Conclusion}

The two scaling relations, one between $M_{0}$ and $f_{0}$ and the other between $M_{0}$ and $E_{\mathrm{R}}$, are not independent. Using the variational calculus, we showed that the ratio $\tilde{e}=E_{\mathrm{R}} /$ $M_{0}$ has a lower bound, $\tilde{e}_{\min }$, for a given $M_{0}$ and $f_{0}$. If the commonly used static scaling relation $M_{0} \propto f_{0}^{-3}$ holds, then $\tilde{e}_{\text {min }}$ must be scale independent and should not depend on $M_{\mathrm{w}}$. The observed values of $\tilde{e}$ for large earthquakes [e.g., $\left.\tilde{e}\left(M_{\mathrm{w}} 7\right)\right]$ are close to $\tilde{e}_{\min }$. The observed values of $\tilde{e}$ for small earthquakes are controversial, but the reported values of $\tilde{e}\left(M_{\mathrm{w}} 3\right)$ range from 1 to 0.1 of $\tilde{e}\left(M_{\mathrm{w}} 7\right)$, suggesting that $\tilde{e}_{\text {min }}$ may decrease as $M_{\mathrm{w}}$ decreases. To accommodate this possibility, we need to modify the $M_{0}$ versus $f_{0}$ scaling relation to $M_{0} \propto f_{0}^{-(3+\varepsilon)}(\varepsilon \leq 1)$, which is allowable within the observational uncertainties. This modification leads to a scaledependent $\tilde{e}_{\min }, \tilde{e}_{\min } \propto 10^{1.5 M_{\mathrm{w}} \varepsilon /(3+\varepsilon)}$, and a scale-dependent $\Delta \sigma_{\mathrm{s}} V^{3}\left(\Delta \sigma_{\mathrm{s}}=\right.$ stress drop, $V=$ rupture speed), $\Delta \sigma_{\mathrm{s}} V^{3} \propto$ $10^{1.5 M_{\mathrm{w}} \varepsilon /(3+\varepsilon)}$, and it can accommodate the range of presently available data on these scaling relations. We note that the scaling relation, $\Delta \sigma_{\mathrm{s}} V^{3} \propto 10^{1.5 M_{\mathrm{w}} \varepsilon /(3+\varepsilon)}$, suggests that even if $\tilde{e}$ is scale independent and $M_{0} \propto f_{0}^{-3}$ (i.e., $\varepsilon=0$ ), $\Delta \sigma_{\mathrm{s}}$ is not necessarily scale independent; such scale independence is often implied. Small and large earthquakes can have significantly different $\Delta \sigma_{\mathrm{s}}$ and $V$; if $\tilde{e}$ varies with $M_{\mathrm{w}}$, as suggested by many data sets, the difference can be even larger. Because $V$ is directly related to the fracture energy, which controls rupture dynamics, this result has an important implication for understanding the physics of earthquakes, and more accurate determinations of $E_{\mathrm{R}}, V$, and $\Delta \sigma_{\mathrm{s}}$ are desired.

\section{Acknowledgments}

We thank Jiakang Xie for reviewing the manuscript and providing helpful comments on the original manuscript.

\section{References}

Abercrombie, R. (1995). Earthquake source scaling relationships from - 1 to $5 M_{\mathrm{L}}$ using seismograms recorded at $2.5-\mathrm{km}$ depth, J. Geophys. Res. 100, 24,015-24,036.

Abercombie, R. E., and P. Leray (1993). Source parameters of small earthquakes recorded at $2.5 \mathrm{~km}$ depth, Cajon Pass, southern California: implications for earthquake scaling, Geophys. Res. Lett. 20, 15111514

Aki, K. (1966). Generation and propagation of $G$ waves from the Niigata earthquake of June 16, 1964. Part 2: Estimation of earthquake moment, from the $G$ wave spectrum, Bull. Earthquake Res. Inst. Tokyo Univ. 44, 73-88.

Beeler, N. M., T.-F. Wong, and S. H. Hickman (2003). On the expected relationships among apparent stress, static stress drop, effective shear fracture energy, and efficiency, Bull. Seism. Soc. Am. 93, 1381-1389.

Boatwright, J. (1994). Regional propagation characteristics and source parameters of earthquakes in northeastern North America, Bull. Seism. Soc. Am. 84, 1-15.

Boatwright, J., and G. L. Choy (1986). Teleseismic estimates of the energy radiated by shallow earthquakes, J. Geophys. Res. 91, 2095-2112.

Boatwright, J., G. L. Choy, and L. C. Seekins (2002). Regional estimates of radiated seismic energy, Bull. Seism. Soc. Am. 92, 1241-1255.

Brune, J. (1970). Tectonic stress and seismic shear waves from earthquakes, J. Geophys. Res. 75, 4997-5009.

Brune, J. (1971). Tectonic stress and seismic shear waves from earthquakes, Correction, J. Geophys. Res. 76, 5002.

Choy, G. L., and J. L. Boatwright (1995). Global patterns of radiated energy and apparent stress, J. Geophys. Res. 100, 18,205-18,228.

Hanks, T. C., and M. Wyss (1972). The use of bodywave spectra in the determination of seismic source parameters, Bull. Seism. Soc. Am. 62, 561-589.

Haskell, N. (1964). Total energy and energy spectral density of elastic wave radiation from propagating faults, Bull. Seism. Soc. Am. 56, 18111842.

Ide, S., and G. C. Beroza (2001). Does apparent stress vary with earthquake size? Geophys. Res. Lett. 28, 3349-3352.

Izutani, Y., and H. Kanamori (2001). Scale dependence of seismic energyto-moment ratio for strike-slip earthquakes in Japan, Geophys. Res. Lett. 28, 4007-4010.

Jost, M. L., T. Büßelberg, Ö. Jost, and H. P. Harjes (1998). Source parameters of injection-induced microearthquakes at $9 \mathrm{~km}$ depth at the KTB deep drilling site, Germany, Bull. Seism. Soc. Am. 88, 815-832.

Kanamori, H., and T. Heaton (2000). Microscopic and macroscopic mechanisms of earthquakes, in GeoComplexity and Physics of Earthquakes, J. Rundle, D. L. Turcotte, and W. Klein (Editors), American Geophysical Monograph 120, 147-163.

Kanamori, H., D. L. Anderson, and T. H. Heaton (1998). Frictional melting during the rupture of the 1994 Bolivian earthquake, Science, 279, 839-842.

Kanamori, H., J. Mori, E. Hauksson, T. H. Heaton, L. K. Hutton, and L. M. Jones (1993). Determination of earthquake energy release and $M_{\mathrm{L}}$ using TERRAscope, Bull. Seism. Soc. Am. 83, 330-346.

Kinoshita, S., and M. Ohike (2002). Scaling relations of earthquakes that occurred in the upper part of the Philippine Sea plate beneath the Kanto region, Japan, estimated by means of borehole recordings, Bull. Seism. Soc. Am. 92, 611-624.

Kostrov, B. V. (1966). Unsteady propagation of longitudinal shear cracks, J. Appl. Math. Mech. 30, 1241-1248.

Mayeda, K., and W. R. Walter (1996). Moment, energy, stress drop, and source spectra of western United States earthquakes from regional coda envelopes, J. Geophys. Res. 101, 11,195-11,208.

McGarr, A., and J. B. Fletcher (2002). Mapping apparent stress and energy radiation over fault zones of major earthquakes, Bull. Seism. Soc. Am. 92, 1633-1646.

Nadeau, R. M., and L. R. Johnson (1998). Seismological studies at Parkfield, part VI: Moment release rates and estimates of source parameters for small repeating earthquakes, Bull. Seism. Soc. Am. 88, 790814.

Pérez-Campos, X., and G. C. Beroza (2001). An apparent mechanism dependence of radiated seismic energy, J. Geophys. Res. 106, 11,12711,136 .

Prejean, S. G., and W. L. Ellsworth (2001). Observations of earthquake source parameters at $2 \mathrm{~km}$ depth in the Long Valey Caldera, eastern California, Bull. Seism. Soc. Am. 91, 165-177.

Richardson, E., and T. H. Jordan (2002). Seismicity in deep gold mines of South Africa: implications for tectonic earthquakes, Bull. Seism. Soc. Am. 92, 1766-1782.

Venkataraman, A., L. Rivera, and H. Kanamori (2002). Radiated energy from the October 16, 1999 Hector Mine earthquake: regional and teleseismic estimates, Bull. Seism. Soc. Am. 92, 1256-1265. 
Wyss, M., and J. N. Brune (1968). Seismic moment, stress, and source dimensions for earthquakes in the California-Nevada region, J. Geophys. Res. 73, 4681-4694.

Xie, J., and H. J. Patton (1999). Regional phase excitation and propagation in the Lop Nor region of central Asia and implications for $P / L g$ discriminants, J. Geophys. Res. 104, 941-954.
Seismological Laboratory

California Institute of Technology

Pasadena, California 91125

(H.K.)

EOST-IPGS

Université Louis Pasteur

5, Rue René Descartes

F67084 Strasbourg, France

(L.R.)

Manuscript received 4 August 2003. 